\title{
LED Based Spectrophotometer can compete with conventional one
}

\author{
Karim Aly Mohammad *, Abdelhalim Zekry, Mohamed Abouelatta \\ Communication Engineering Department, Faculty of Engineering, Ain shams University, Egypt \\ *Corresponding author E-mail: Karimaly08@gmail.com
}

Copyright () 2015 Karim Aly Mohammad et al. This is an open access article distributed under the Creative Commons Attribution License, which permits unrestricted use, distribution, and reproduction in any medium, provided the original work is properly cited.

\begin{abstract}
Spectrophotometers are the common devise used for blood analysis. These analyses are simply optioned from the transmittance of monochromatic light through a blood sample. It disperses their light sources by different techniques; the simplest one is the use of interference filters in front of broad-band light source. As Interference bandpass filters are relatively inexpensive wavelength selectors. Interference filters that allow transmission of a predetermined wavelength while rejecting or blocking other wavelengths are widely used in instrumentation for clinical chemistry. On the other hand, the lights emitting diodes (LEDs) emits a specified band and have a wide selection options. An economic device based on LEDs (LED based spectrometer) is explained in this work. Instead of the wide spectrum light source and filters (commercial spectrophotometer), The LED based spectrophotometer is calibrated with standard solutions, and its measurement results is compared with the Mindray BS-200 Chemistry Analyzer (commercial spectrophotometer). The comparison shows acceptable results between both spectrophotometers with maximum error $2.33 \%$. The errors can be reduced by applying more fining alignment. Another benefit of the new designee is to minimize testing costs.
\end{abstract}

Keywords: LED; Spectrophotometer; Mindray BS-200; Blood Analysis; Chemical Analysis.

\section{Introduction}

A spectrophotometer is defined as an instrument for measuring the transmittance or absorbance of a sample as a function of the wavelength of electromagnetic radiation [1].

The key components of the traditional spectrophotometer are [1]:

1) A source that emits a broad band of electromagnetic radiation.

2) A dispersion device that selects a particular wavelength (or, more correctly, a waveband) from the broadband radiation of the source.

3) A sample area.

4) One or more detectors to measure the intensity of radiation.

Reliance on the use of LEDs imposes itself in this era, with the development of scientific and technical properties of these LEDs. They spread dramatically with many advantages of their small size, long life and relatively high efficacy. The availability of wide selection of significant wavelengths, hence, the researchers used them in many applications, including reliable hardware configuration spectrophotometer. Hauser et al.[2-4] were one of the first researchers who constructed a LED based spectrophotometer using 7 LEDs with different wavelengths to analyze aluminum, copper, ammonia, calcium, phosphate, chromium, and nitrite as a Qualitative measurement device. A Flow-Injection analysis using a Tri-Colour LEDs as a LED based spectrophotometers was reviewed by Nataša Gros [5]. Degner et al. [6], [7] presented a low-cost high resolution (ppm and ppb range) LED based spectrometric sensor system for gas detection in harsh environment. This method is suitable almost for substances with broadband absorption characteristics. For substances with narrow band absorption characteristics, they innovated a new method based on Multi Quantum Well LEDs. Yeh and Tsnag [8] constructed a LED based spectrophotometer for qualitative and quantitative measurements and have good results but for absorbance values under 0.8 . 
In this paper, it is intended to build a complete standalone LED based spectrometer device for special factor analysis applications with high accuracy compared with other commercial LAB devices.

\section{Conventional spectrophotometer}

A Polychromatic light from light source is focused upon the entrance slit of a monochromator, which selectively transmits a narrow band of light. This light then passes through the sample area to the detector as shown in Fig. 1.

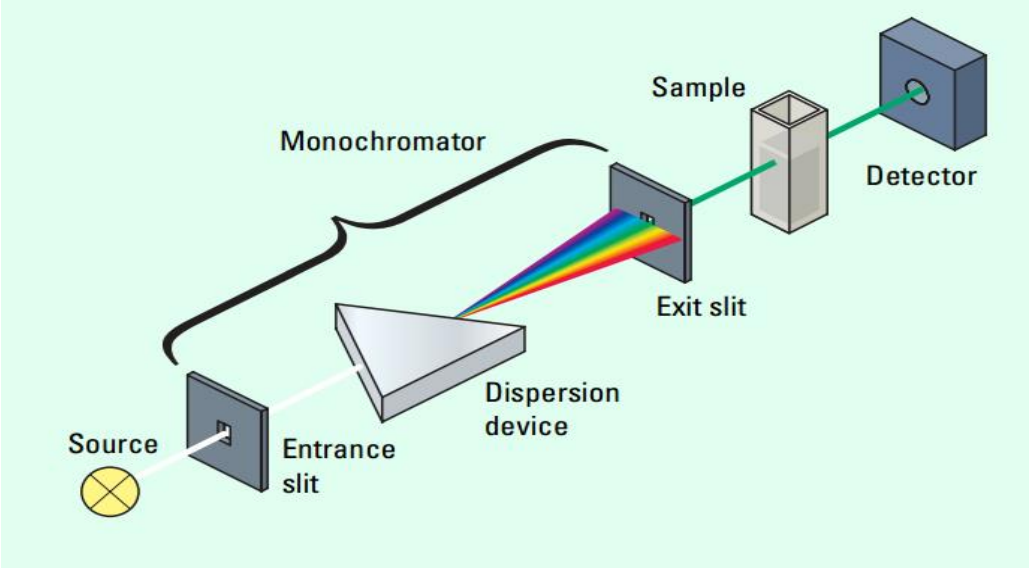

Fig. 1: Sketch of Conventional Spectrophotometer System [1].

The absorbance of a sample is determined by measuring the intensity of light reaching the detector without the sample (through the reagent blank) and comparing it with the intensity of light reaching the detector transmitted through the sample.

The relation between transmittance $(\mathrm{T})$ and concentration $(\mathrm{C})$ is determined through Beer -Lambert law, which states that [1]:

$$
T=I / I_{0}=e^{k b C}, A=-\log T=-\log \left(I / I_{0}\right)=\varepsilon b C
$$

Where Io is the incident intensity, $\mathrm{I}$ is the transmitted intensity, e is the base of natural logarithms; $\mathrm{k}$ is a constant, $\mathrm{b}$ is the path length (usually in centimeters), $\mathrm{C}$ is the concentration of the absorbing species (usually expressed in grams per liter or milligrams per liter), and $\mathrm{A}$ is the Absorbance [1].

The exponential relation between the transmittance and the concentration is depicted in Fig. 2, While, the relation between the absorbance, and the Concentration is a linear function as shown in Fig. 3.

The Concentration can then be obtained from the slope of the straight line as $\mathrm{C}=\mathrm{A} / \mathrm{\varepsilon b}$.

The new economic instrument is based on LEDs as monochromatic substituting the broad-band source and dispersion device through which particular wavelength can be selected from the broadband radiation of the source in the commercial spectrophotometer.

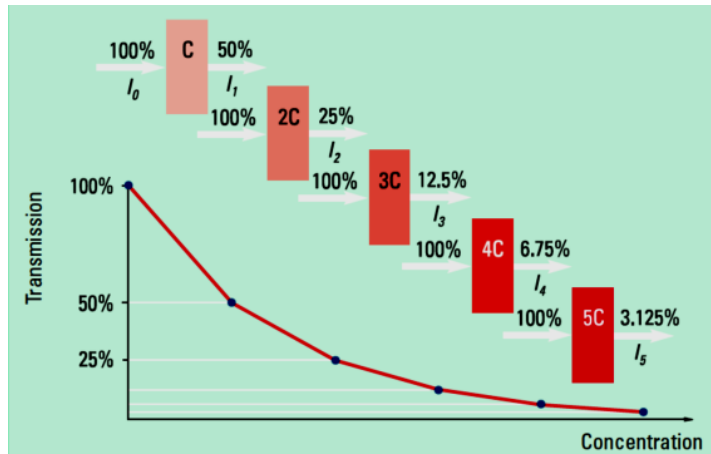

Fig. 2: The Exponential Relation between the Transmittance and the Concentration [1].

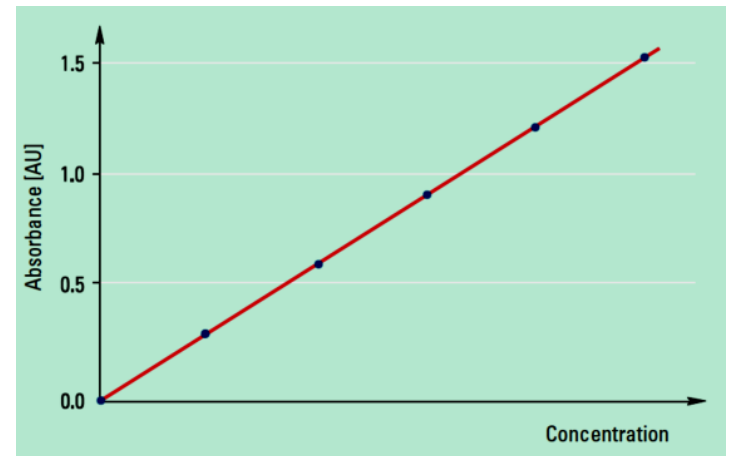

Fig. 3: The Linear Relation between the Absorbance and the Concentration [1].

\section{Materials and methods}

In this section, a detailed description of the design, implementation, testing and calibration of the new spectrophotometer based on using LEDS as monochromatic light sources is introduced. 


\subsection{The chemical reagent and electronic components}

The chemical reagent of Glucose and its standard calibration solution [9] - from Linear Chemicals Company - are used for calibrating the new device and measure the Glucose concentrations in some blood serums.

The light sources utilized are commercial LEDs that have as narrow radiation band width as possible. The implemented tester depends on using HLMP-CE35-Y1CDD [10] Cyan LED, which has a Dominant Wavelength $=505 \mathrm{~nm}$ (the same wavelength required for the glucose test) and spectral half width $=30 \mathrm{~nm}$. The LED light intensity can by adjusted by a $2 \mathrm{~K} \Omega$ variable resistor.

The programmable light-to-frequency transducer chip TCS3200 [11] is used as a photo detector which converts the light intensity to frequency, a Graphical LCD (GLCD) module is used to display the measured data of the photo detector. The system is powered by a simple 9 DCV adapter. Other discrete components are used for interface and power handling and will be determined in the next sections.

\subsection{System block diagram}

The system block diagram is shown in Fig. 4. It consists of four parts: A 9 DCV adapter, A GLCD displays module, interface board, and set of different waveband emitting LEDs and TCS3200 printed circuit boards.

First part: A 9 DCV adapter, which supply the electronic circuit with the appropriate power.

Second part: A GLCD video display module which contains a monochromatic $240 * 128$ GLCD, and a PIC18F8722 microcontroller board. The PIC18F8722 is programmed with the miKroC PRO program. It reads the output frequency of the photodetector, converts it to the corresponding glucose concentration value using the calibration equation as will be described, and then display this result on the GLCD screen.

Third part: Interface board shown in Fig. 5, which acts as a mediator between the PIC18F8722, the LEDs and TCS3200 boards. From the figure, its main contents are:

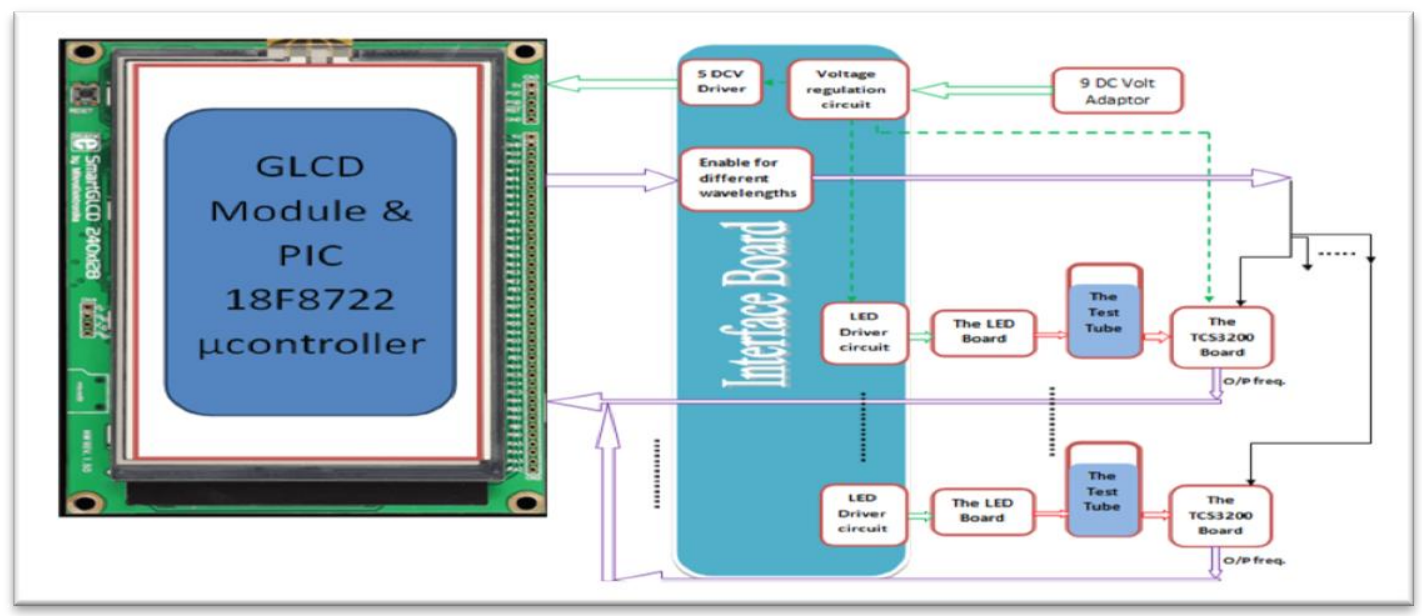

Fig. 4: The LED Based Spectrophotometer Block Diagram.

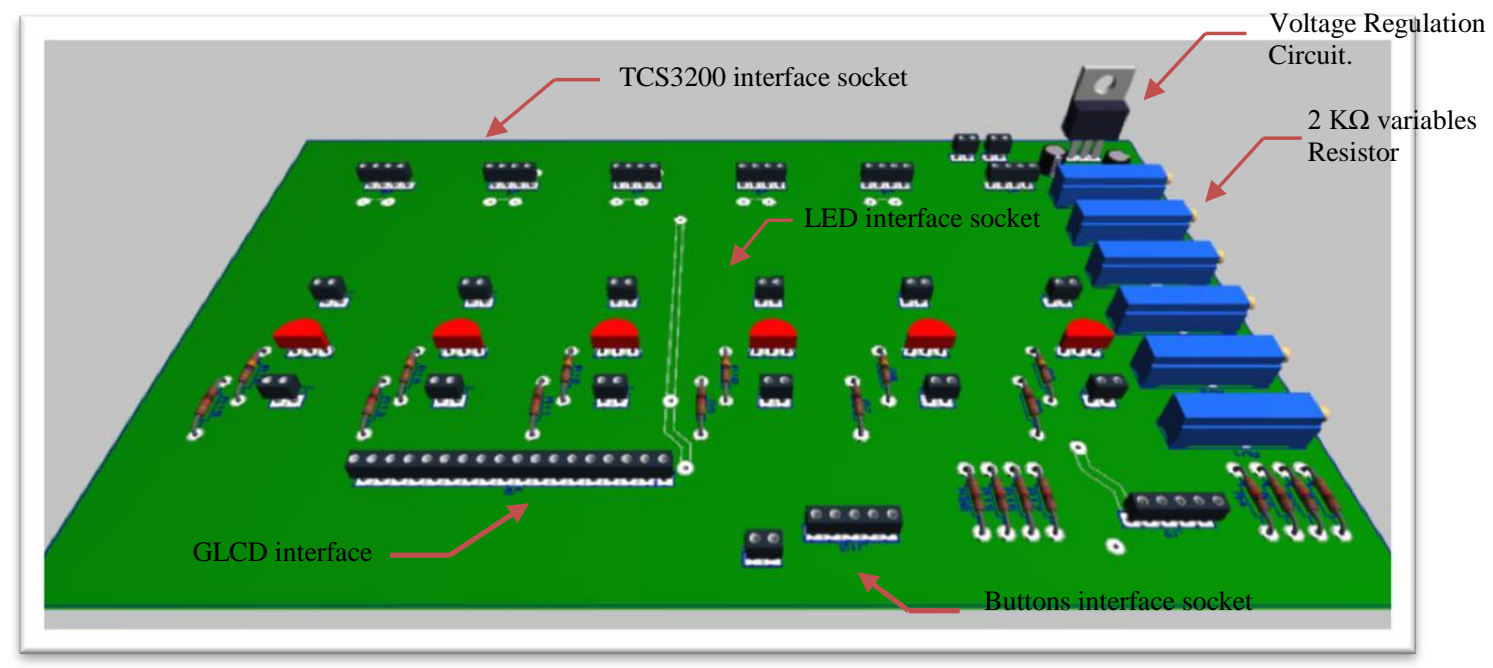

Fig. 5: 3D Model of the Interface Board Layout. 
a) Voltage regulation circuit as depicted in Fig. 6, which steps down the input adaptor voltage to the appropriate voltage rating to supply the electronic components.

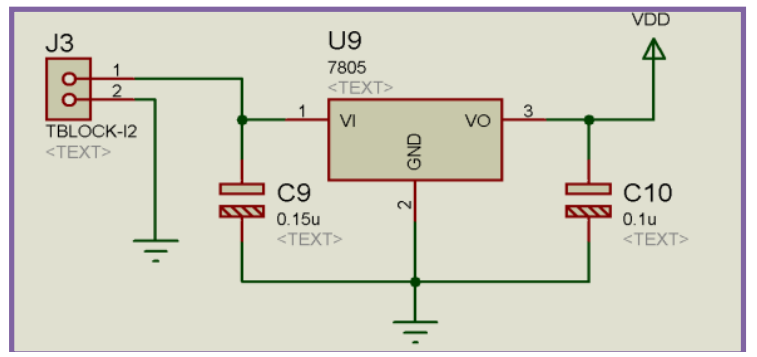

Fig. 6: Voltage Regulation Circuit of the Interface Board.

b) LEDs driver circuits as depicted in Fig. 7, which controls the LED light intensity through the 2 K $\Omega$ resistors.

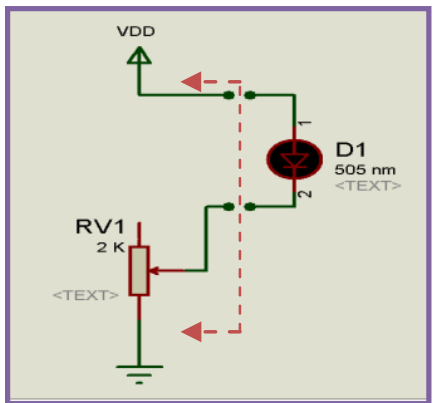

Fig. 7: LED Driver Circuit of the Interface Board.

c) GLCD interface socket which handles the microcontroller Enable signals to the TCS3200 boards and handle its output frequency to the microcontroller again.

d) TCS3200 interface socket with 4 pins, which supply the chip with the required power, Enable signal and readout the chip output frequency to handle it to the GLCD interface socket.

Fourth: Set of different waveband emitting LEDs and TCS3200 photo detector printed circuit boards (PCBs) as shown in Fig. 8 and Fig. 9, which assembled in a round shapes to be fitted in the optical system holders.

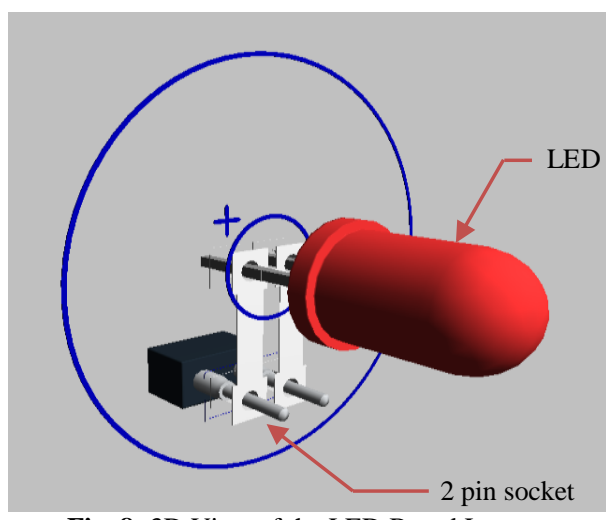

Fig. 8: 3D View of the LED Board Layout.

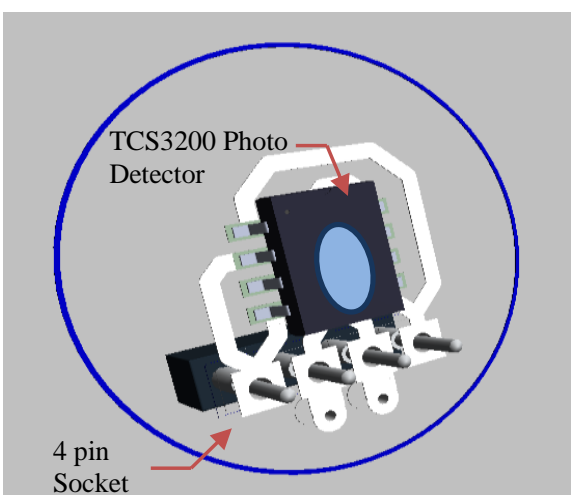

Fig. 9: 3D View of the TCS3200 Board Layout. 


\section{The optical system}

The whole optical system of the spectrophotometer is indicated in Fig. 10 and Fig. 11. It consists of the parts:

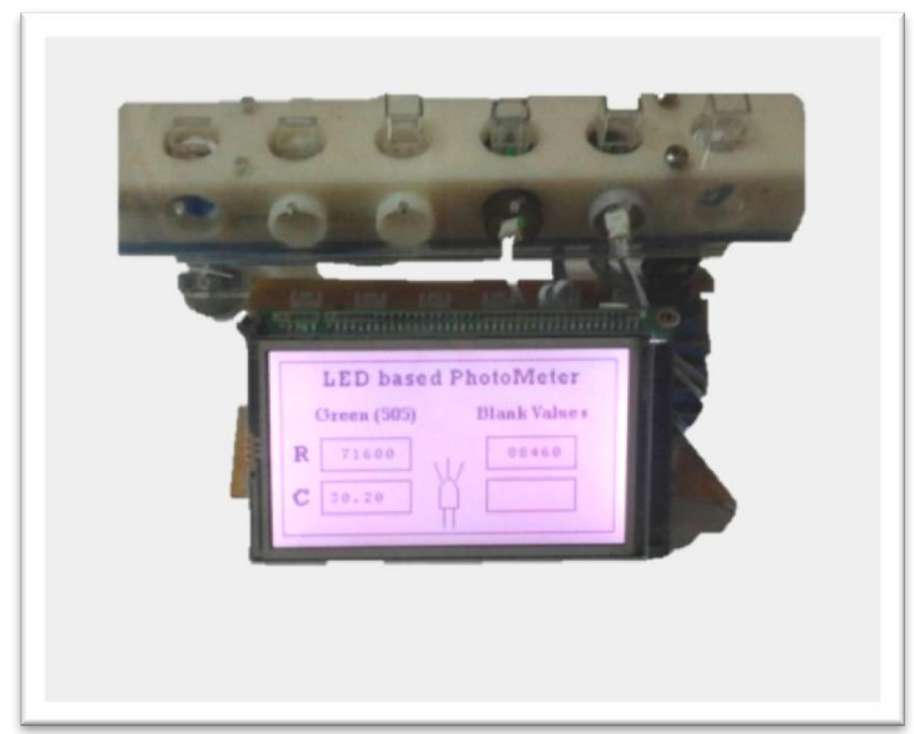

Fig. 10: The LED Based Spectrophotometer Device.

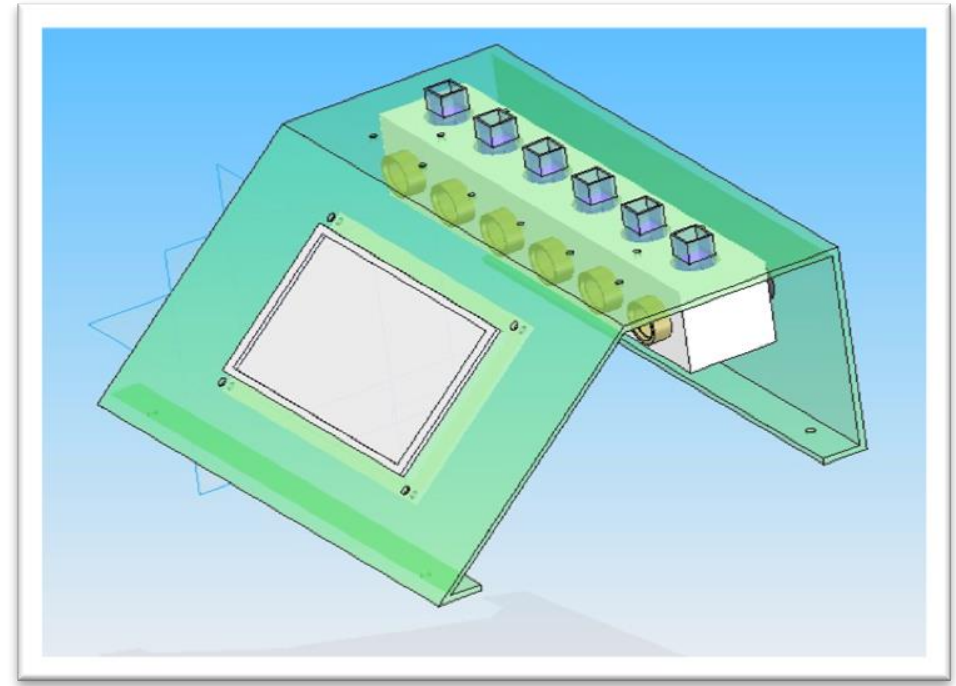

Fig. 11: 3D Model of the LED Based Spectrophotometer.

The optical holder: It consists of 6 channels as shown in Fig. 12 where each channel holds an optical measurement system. The channel simply is a cube having two perpendiculars through holes.

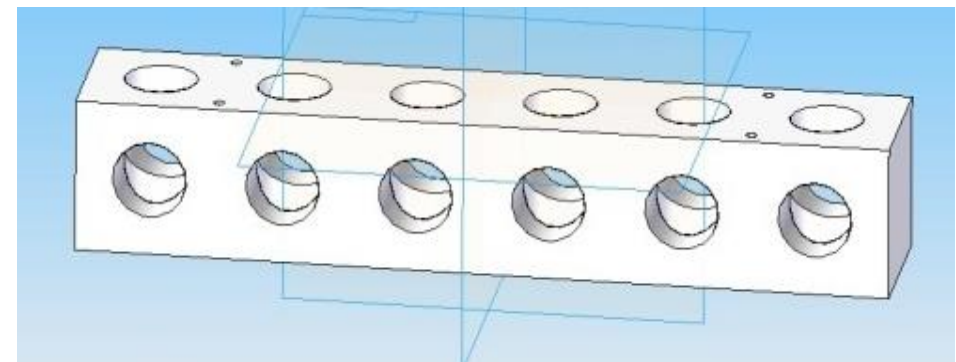

Fig. 12: The Optical Holder.

The LED holder: It holds the LED board in its cavity ending with a small through the hole opposite to the center of the LED as shown in Fig. 13. The LED light emanates from this hole and passes through the test tube. 


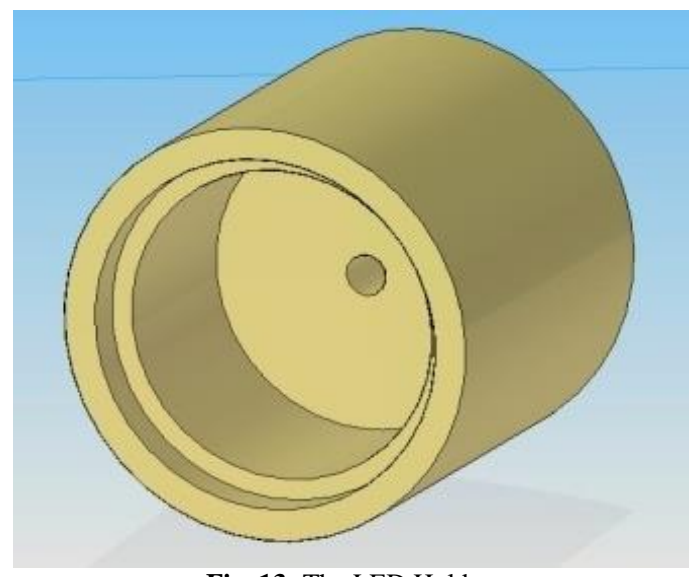

Fig. 13: The LED Holder.

The photo detector holder: holds the TCS3200 photodetecor chip board in its cavity ending with a through the hole in the side of the exposed surface of the detector. Its diameter is made larger than the detector surface area as shown in Fig. 14.

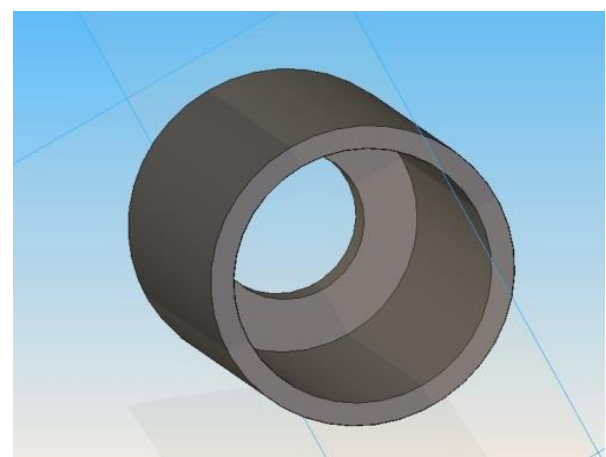

Fig. 14: The Photo Detector Holder.

The measurement tube: It is a standard test tube containing the test sample as shown in Fig. 15, and slides through the vertical optical holder channels.

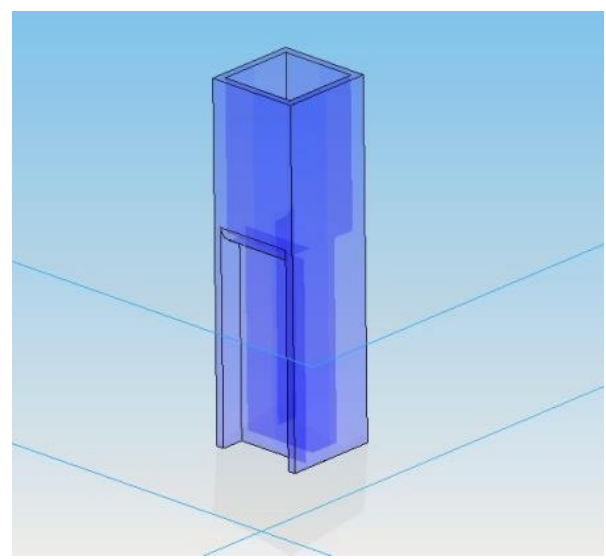

Fig. 15: The Measurement Tube.

As demonstrated in Fig. 16 and Fig. 17, the main function of the optical system is aligning the LED, the photodetector and the measurement tube to be at the same center line. 


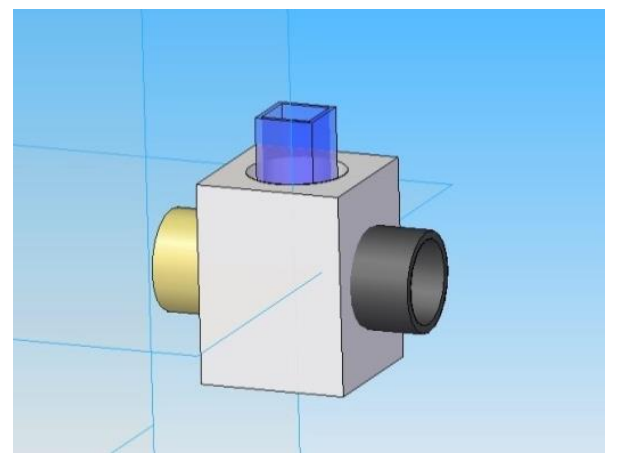

Fig. 16: The Assembled Optical Measurement System.

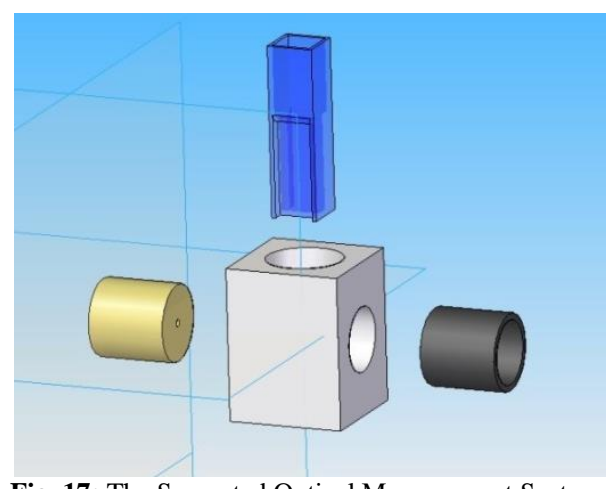

Fig. 17: The Separated Optical Measurement System.

All components of the optical system are painted with black color to decrease the stray light. The system has no lenses to focus the LED light, but depends on the small hole located in the LED holder.

\section{The device calibration}

After finishing the production of the photo spectrometer, it has been calibrated. The calibration is based on measuring known values of Glucose concentration samples and read out the detector value each time, then getting out the relation between the detector readings and the Glucose concentrations. Using the Glucose reagent and its calibration solution, we can prepare the required concentrations for the calibration process.

The prepared glucose concentrations are: zero mg/dl (reagent blank), $100 \mathrm{mg} / \mathrm{dl}, 200 \mathrm{mg} / \mathrm{dl}, 300 \mathrm{mg} / \mathrm{dl}$ and $400 \mathrm{mg} / \mathrm{dl}$. Starting the test with zero $\mathrm{mg} / \mathrm{dl}$ (reagent blank) solution, the measurement tube is centered between the LED source and the detector by putting it in the device tube holder channel. The detector reading is adjusted by varying the light intensity of the LED using the $2 \mathrm{k} \Omega$ Variable resistor until the detector reads a frequency of $133.500 \mathrm{KHz}$, which corresponds to the transmitted light with zero glucose concentration. This value will be the zero point of calibration process. To obtain the detector read out for the reaming concentrations, other test tubes filled with the required concentrations are inserted and the corresponding readings of the photo detector ar place in the device and recording the read out. The obtained test results are shown in table 1.

Table 1: Calibration Data of the Produced Spectrophotometer

\begin{tabular}{ll}
\hline Concentration $(\mathrm{mg} / \mathrm{dl})$ & Detector readout $(\mathrm{Hz})$ \\
\hline 0 & 133500 \\
100 & 64680 \\
200 & 32000 \\
300 & 15020 \\
400 & 7180 \\
\hline
\end{tabular}

The obtained calibration results are plotted in a semi logarithmic and curve fitted by the equation,

$\ln (f)=\ln \left(f_{0}\right)+m \times C$

Where $\mathrm{f}$ is considered proportional to the light intensity I. The parameters of the curve fitting are:

Io $=133500$, and $\mathrm{m}=-0.007$. 
The Glucose concentration can then be calculated using the final formula:

$C=\ln (f / f 0) / 0.007$

Equation 3 is called the calibration equation and is used by to the device software to calculate the concentration corresponding to nay frequency read out. The concentration is then displayed directly on the GLCD screen.

The flow chart of the automatic test and display procedure implemented by the microcontroller is depicted in Fig. 18.

\section{Test procedure}

After the calibration of the instrument. It is used to measure glucose concentration in real blood serums in standard test laboratories. The same specimens are measured at the same time using the Mindray BS-200 as a standard test instrument. The experimental results are shown in table 2.

Table 2: Field Test Results of Our Instrument and Mindray BS-200

\begin{tabular}{llll}
\hline Serum number & Mindray BS200 readings (mg/dl) & $\begin{array}{l}\text { LED Based Spectrophotometer readings } \\
(\mathrm{mg} / \mathrm{dl})\end{array}$ & $\%$ Error \\
\hline 1 & 91 & 88.88 & -2.33 \\
2 & 100 & 99 & -1 \\
3 & 200 & 198.3 & -0.85 \\
4 & 261 & 259.2 & -0.69 \\
5 & 349 & 347.5 & -0.43 \\
\hline
\end{tabular}

The \% errors of the LED based spectrophotometer are calculated as:

$\%$ Error $=\frac{\text { LED based spectrophotometer reading }- \text { Mindray BS-200 reading }}{\text { Mindray BS-200 reading }} * 100$

It is seen from the table that max error is $-2.33 \%$, which is considered very acceptable.

\section{Conclusions}

A construction of new cheap and accurate instruments demanded in our life, but it needs huge efforts to implement it. Our instrument is an example where one can make full use of the new devices and components to develop wellfunctioning instruments that can substitute the classical instruments and setups. It has been shown throughout this work the new LED based spectrophotometer can perform the same function of the traditional spectrophotometer devices and achieve the same accurate results.

The constructed LED spectrophotometer has the advantage of low power consumption, low price, simple design, relatively long life stable monochromatic sources, and acceptable results as that obtained from the existing ones of more sophisticated and expensive components.

The developed spectrophotometer can be improved by:

1) Add optical focusing system to achieve parallel beam.

2) Fining the measurement tube fittings mechanism to be at the same position in each reading process and this is a large source of error.

The LED source is derived from a simple circuit of a DC Voltage source and series resistance with the LED source; any change in voltage or resistor value will change the output light intensity, the best way of driving the LEDs is a controlled current source, which independent on the DC voltage source variations and can be controlled by the microcontroller. 


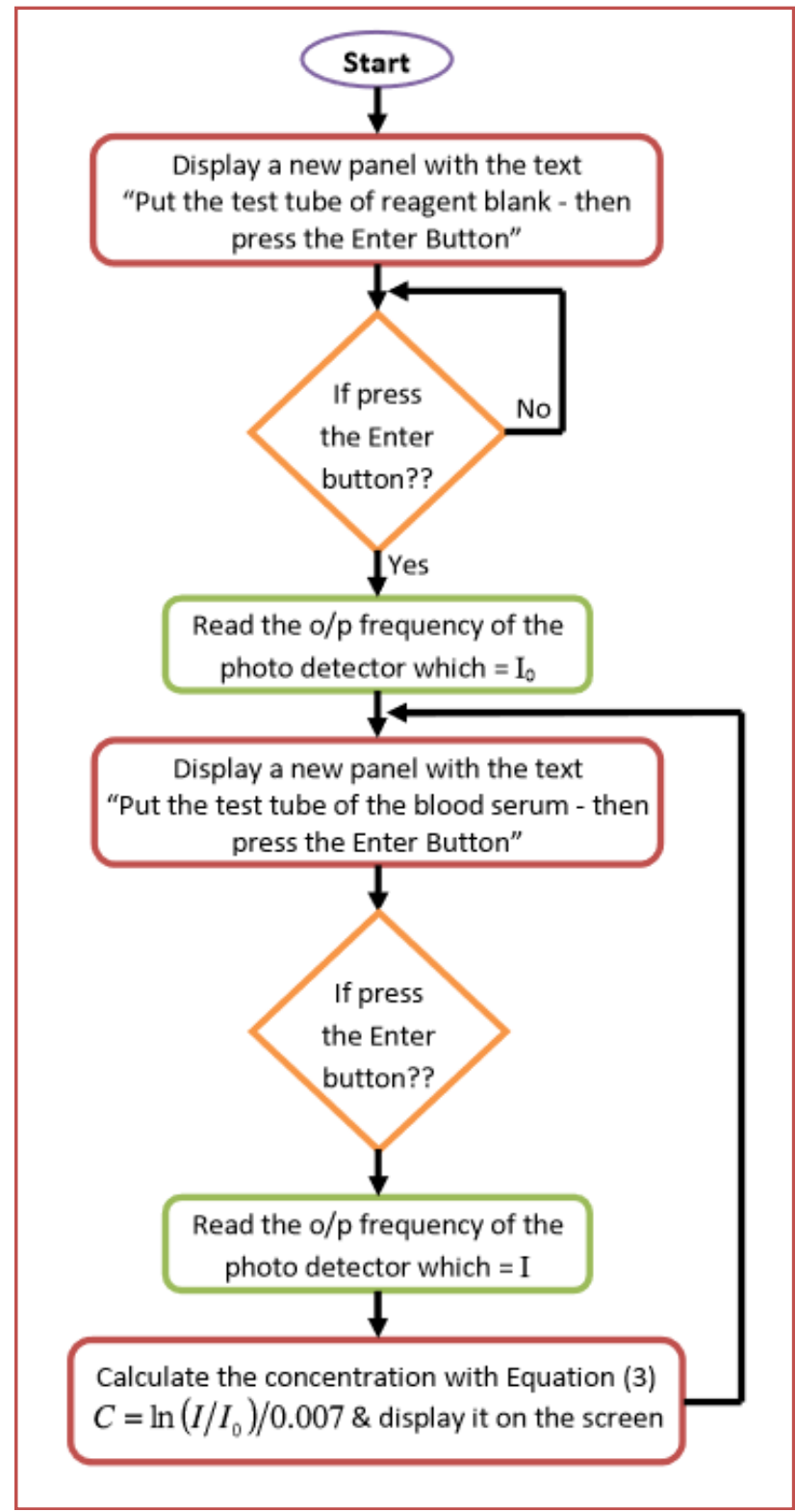

Fig. 18: Flow Chart of the LED Based Spectrophotometer Software.

\section{References}

[1] Tony Owen, "Fundamentals of modern UV-visible spectroscopy", Agilent Technologies, Germany, 2000.

[2] Peter C. Hauser, Thusitha W. T. Rupasinghe and Norman E. Cates, "A multi-wavelength photometer based on light-emitting diodes", Talanta, vol. 42, pp. 605-612, 1995. http://dx.doi.org/10.1016/0039-9140(95)01455-K.

[3] Peter C. Hauser, Thusitha W. T. Rupasinghe and R. Tan, "Simultaneous determination of metal ion concentrations in binary mixtures with a multi-LED photometer", Chimia, vol. 49, pp. 492-494, 1995.

[4] Peter C. Hauser and Thusitha W. T. Rupasinghe, "Simultaneous determination of metal ion concentrations in binary mixtures with a multiLED photometer", Fresenius Journal of Analytical Chemistry, vol. 357, pp. 1056-1060, 1997. http://dx.doi.org/10.1007/s002160050304.

[5] Nataša Gros, "A Novel Type of Tri-Colour Light-Emitting-Diode-Based Spectrometric Detector for Low-Budget Flow-Injection Analysis", Sensors journal, vol. 7, pp. 166-184, 2007.

[6] M. Degner, H. Ewald and E. Lewis, "LED Based Spectroscopy - a Low Cost Solution for High Resolution Concentration Measurements e.g. for Gas Monitoring Applications", Fifth International Conference on Sensing Technology (ICST), pp. 145 - 150 , 2011. http://dx.doi.org/10.1109/ICSensT.2011.6136951.

[7] M. Degner and H. Ewald, "LED-Spectroscopy based on Multi Quantum Well Emitter", International Conference on Electromagnetics in Advanced Applications (ICEAA), pp. 840 - 843, 2012. http://dx.doi.org/10.1109/ICEAA.2012.6328751.

[8] Tai-Sheng Yeh and Shih-Shin Tseng, "A low cost LED based spectrometer", Journal of the Chinese Chemical Society, vol. 53, pp. 1067-1072, 2006. http://dx.doi.org/10.1002/jccs.200600142.

[9] http://www.linear.es/ficheros/archivos/411129005I.pdf.March 2015.

[10] http://www.avagotech.com/pages/en/leds/high_brightness_through_hole_lamps/round_5mm_white/hlmp-cw47-qrb00/.

[11] http://ams.com/eng/Products/Light-Sensors/Color-Sensor/TCS3200. 